\title{
Detection of spectroscopic binaries: lessons from the Gaia-ESO survey
}

\author{
Mathieu Van der Swaelmen ${ }^{1}$, Thibault Merle ${ }^{1}$, Sophie Van Eck ${ }^{1}$, \\ Alain Jorissen ${ }^{1}$ and Tomaž Zwitter ${ }^{2}$ \\ ${ }^{1}$ Institut d'Astronomie et d'Astrophysique, Université Libre de Bruxelles, Belgium \\ email: mathieu.van.der.swaelmen@ulb.ac.be \\ ${ }^{2}$ Faculty of Mathematics and Physics, University of Ljubljana, Slovenia
}

\begin{abstract}
The Gaia-ESO survey (GES; Gilmore et al. (2012), Randich et al. (2013)) is a spectroscopic survey complementing the Gaia mission to bring accurate radial velocities and chemical abundances for $10^{5}$ stars. Merle et al. (submitted to A\&A; see also this volume) developped a tool (DOE) to detect multiple peaks in the cross-correlation functions (CCFs) of GES spectra. Using the GIRAFFE HR10 and HR21 settings, we were able to compare the efficiency of our SB detection tool depending on the wavelength range and resolution. We show that a careful design of CCF masks can improve the detection rate in the HR21 settings. HR21 spectra are similar to the ones produced by the RVS spectrograph of the Gaia mission, though the lower resolution of RVS spectra may result in a lower detection efficiency than the case of HR21. Analysis of RVS spectra in the context of spectroscopic binaries can take advantage of the lessons learnt from the GES to maximize the detection rate.
\end{abstract}

Keywords. binaries: spectroscopic, techniques: radial velocities, techniques: spectroscopic, surveys, catalogs

\section{Methods \& Results}

We run Monte-Carlo simulations to generate HR10 and HR21 spectra $(R \sim 21500$ and $R \sim 18000$, resp.) of a pair of twin (non-rotating) stars for various levels of $\mathrm{S} / \mathrm{N}$ and radial velocity separations $\Delta v_{\text {rad }}$. We then apply our DOE pipeline on the simulated spectra. Fig. 1 shows the SB2 detection efficiency in HR10 and HR21. The green dots (respectively the red triangles) indicate $\left(\Delta v_{\mathrm{rad}}, \mathrm{S} / \mathrm{N}\right)$ conditions when DOE is able to detect the two expected peaks in more than $95 \%$ of cases (resp., conditions when DOE failed at detecting two expected peaks in more than $95 \%$ of cases). Blue plusses represent intermediate cases making detection efficiency dependent of the noise: (i) due to the noise, spurious peaks may appear or (ii) thanks to the noise, the two peaks have different height (despite being a pair of twins) and become discernible to DOE for small $\Delta v_{\text {rad }}$. Our simulations show that HR10 allows a more efficient detection, with a good detection rate as soon as $\mathrm{S} / \mathrm{N} \geqslant 2$ and $\Delta v_{\text {rad }} \geqslant 25 \mathrm{~km} \mathrm{~s}^{-1}$. On the other hand, HR21 allows the detection of SB2 with $\Delta v_{\text {rad }} \geqslant 35 \mathrm{~km} \mathrm{~s}^{-1}$ with $\mathrm{S} / \mathrm{N} \gtrsim 10$. Merle et al. performed a full analysis of GES DR4 spectra looking for stellar multiplicity and their smallest detected $\Delta v_{\text {rad }}$ is $25 \mathrm{~km} \mathrm{~s}^{-1}$ for HR10 and $60 \mathrm{~km} \mathrm{~s}^{-1}$ for HR21, well in line with our predictions.

In order to improve the HR10 and HR21 CCFs, we selected a set of weakly-blended lines in the range $[5330 \AA, 5610 \AA]$ and $[8430 \AA, 8990 \AA]$ and used them to compute synthetic spectra. Fig. 2 compares the GES/CASU CCF (old) to our new CCF for the object 07272578-0310066. While the binary nature was already obvious in HR10 with the GES $\mathrm{CCF}$, it was not detectable in HR21 with the GES CCF. As expected, our new CCFs give the same results for HR10, showing that the method is robust and that our new 

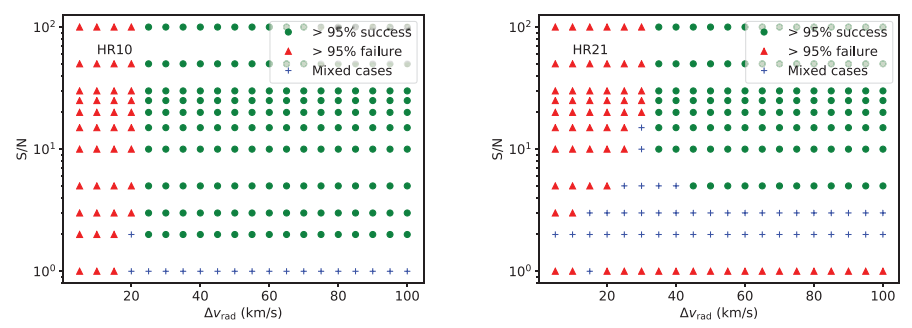

Figure 1. Detection efficiency for HR10 and HR21
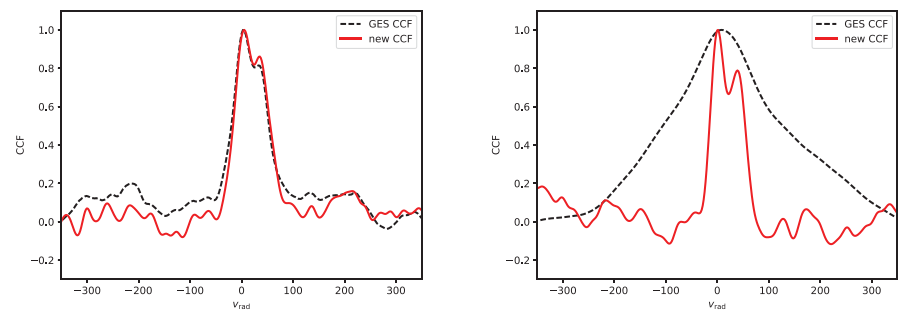

Figure 2. HR10 (left) and HR21 (right) CCF of 07272578-0310066 at MJD 57032.153726 and 57032.247332 (resp.). Black dashed curve is the GES/CASU CCF, red solid curve is the newly computed CCF.

mask allows to retrieve already known SB2s. However, unlike the GES CCF, the new HR21 CCF has narrower peaks and now also reveals the SB2 nature. The broad profile of the HR21 GES CCF is due to the presence of strong lines (Ca II triplet, strong Mg line, Paschen lines) in the range [8430 $\mathrm{A}, 8990 \AA]$. Since our masks do not include such lines, we get narrower CCFs. We performed preliminary tests on a subset of SB2s identified by Merle et al. for which the SB2 nature is detected in HR10 GES CCFs but not in HR21 ones. Our new CCFs now allow to detect the SB2 nature of $\sim 35 \%$ objects based on their HR21 CCFs (26 objects out of 72).

\section{Conclusion}

After applying this analysis to the whole GES survey, we expect to improve our SB catalogue: 1 / by completing the time series of velocity measurements for already known objects (when HR21 did not previously lead to SB detection); 2/ by detecting new SBs among the objects observed only with the HR21 setup; 3/ by detecting new SBs among the objects for which HR10 CCFs did not lead to SB detection (very low S/N, phase not favourable to see all components, etc.). Our work shows that very low $\mathrm{S} / \mathrm{N}$ spectra $(>2$ for HR10; $>5$ for HR21) are still usable in the context of radial velocity measurement and thus, for SB detection. Since the HR21 spectral domain resembles that of the Gaia RVS, RVS CCFs may suffer from similar broadening issues and may benefit from a careful design of correlating masks.

\section{References}

Gilmore, G., Randich, S. et al. 2012, The Messenger, 25, 147

Merle, T., Van Eck, S., Jorissen, A., Van der Swaelmen, M. et al. 2017, A\&A, submitted Randich, S., Gilmore, G., \& Gaia-ESO Consortium 2013, The Messenger, 47, 154 